Relations industrielles

Industrial Relations

\title{
Yannakourou, Stamatina, L'État, l'autonomie collective et le travailleur - Étude comparée du droit italien et du droit français de la représentativité syndicale
}

\section{Pierre Verge}

Volume 52, numéro 3, 1997

URI : https://id.erudit.org/iderudit/051195ar

DOI : https://doi.org/10.7202/051195ar

Aller au sommaire du numéro

Éditeur(s)

Département des relations industrielles de l'Université Laval

ISSN

0034-379X (imprimé)

1703-8138 (numérique)

Découvrir la revue

Citer ce compte rendu

Verge, P. (1997). Compte rendu de [Yannakourou, Stamatina, L'État, l'autonomie collective et le travailleur - Étude comparée du droit italien et du droit français de la représentativité syndicale]. Relations industrielles / Industrial Relations, 52(3), 662-664. https://doi.org/10.7202/051195ar

Tous droits réservés (C) Département des relations industrielles de l'Université Laval, 1997
Ce document est protégé par la loi sur le droit d'auteur. L’utilisation des services d'Érudit (y compris la reproduction) est assujettie à sa politique d'utilisation que vous pouvez consulter en ligne.

https://apropos.erudit.org/fr/usagers/politique-dutilisation/ 
and ironic that this occurred even as the founding president of the union lay buried in the graveyard of St. Anne's Church where Rice served as pastor during this time of estrangement. It was a Canadian, Lynn Williams, who rebuilt the relationship between Phil Murray's union and Phil Murray's friend.

The book is edited with loving care by Dr. Charles McCollester. While the editor is obviously a great admirer of Msgr. Rice, the editing is done with scholarly objectivity. Although the editor provides an historical and biographical framework, primarily he lets Rice speaks for himself. The writings are drawn mainly from a weekly column which Rice wrote over a period of sixty years in the Pittsburgh Catholic (with a several year hiatus when the incumbent Bishop took umbrage at the Monsignor's column critical of a Cardinal for using seminarians as scabs during a gravediggers' strike). Accompanying the writings are a collection of photos of Rice living out his beliefs which by themselves are worth the price of the book $(\$ 49.95$, hardcover; $\$ 19.95$, soft). Buy it, you'll like it!
The flavour of the Rice writings - both content and style - is reflected in the titles of his columns. A few examples will have to suffice: The Irish Revolt, The Dynamite of the Encyclicals, Can a Catholic Accept the Outstretched Hand of a Communist?, Catholic Contempt of the Negro Must End, Catholics and AntiSemitism, Westinghouse and Reds Again, A Company Union Finally Succceeds in Standing on Its Own Feet, How to DeControl Your Union of Communists, The Struggle for UE, The Tragic Purge of 1948, Confessions of an Anti-Communist, Do We Belong in Vietnam, Reuther - R.I.P., Jock Yablonski the Rebel, Nuns and Unions, Mighty Steel Strikes Us Out, We Need a Two - Way Class War, Persian Gulf War Is Not Just, It Is Not Burning that Dishonors Flag, Father Aristede Is My Kind of Guy, Airline Pilots Versus Predators, Steve Nelson: An Honorable Antagonist, The Lord Hears the Cry of the Poor! So Should We.

MARTIN MORAN

Indiana University of Pennsylvania

\section{L'État, l'autonomie collective et le travailleur - Etude comparée du droit italien et du droit français de la représentativité syndicale}

par Stamatina YANNAKOUROU, Bibliothèque du droit privé, tome 247, Paris:

L.G.D.J., 1995, 393 p., ISBN 2-275-00265-0.

Comment une étude comparée, version remaniée d'une thèse de doctorat, portant sur deux régimes juridiques de représentativité syndicale a priori si éloignés de la formule nord-américaine les régimes français et italiens -, peutelle interpeller d'une façon significative le juriste ou le spécialiste des relations industrielles dont le quotidien le rattache à ce dernier modèle?

En premier lieu, l'étude dégage les rapports fondamentaux, les "modes de communication ", entre la négociation, ou mieux, l'autonomie collective et la loi, l'ordre juridique étatique. Le choix des systèmes confrontés assure déjà la fécon- dité de la comparaison : l'Italie " est l'unique pays parmi les démocraties de l'Europe occidentale qui ignore en principe la réglementation législative des rapports de travail " (p. 1) ; l'encadrement législatif des syndicats et de la négociation collective est, au contraire, prononcé en France. L'exposé développement historique de l'un et l'autre systèmes et l'analyse détaillée des apports législatifs, jurisprudentiels et doctrinaux qui les ont façonnés permettent de dégager avec force et netteté leurs orientations plutôt divergentes.

La trame est celle du pluralisme juridique dont est imprégnée la culture 
juridique italienne de l'après-guerre, mais qui est rébarbatif pour le juriste qui adhère au "dogme républicain de la souveraineté nationale et [à] la tradition du droit positif soumis à la loi écrite et incarné par le pouvoir politique" (p.21). En Italie, l'autonomie collective apparait essentiellement comme un "espace de liberté ", "générateur de normes dont la juridicité est indépendante de leur qualification par l'ordre étatique " (p. 62). Une lecture du droit positif français fait voir, au contraire, une loi attributive "d'un pouvoir de réglementation au profit de la collectivité professionnelle " aux acteurs syndicaux (p. 81 et suivantes). La notion de représentativité, appliquée au contexte syndical assure, précisément, la liaison entre le monde syndical et son activité et l'ordre étatique.

Italienne, la représentativité est effective et pragmatique; elle se dégage des rapports de force entre acteur intersyndicaux (p. 177). Elle est, certes, attributive d'un statut au syndicat, mais ne l'habilite pas pour autant "à exercer une activité normative, puisque cette activité relève exclusivement de l'autonomie collective, une sphère normative sur laquelle la loi a peu d'emprise " (p. 181). Dans une perspective néocorporatiste, l'État veut s'assurer de groupements responsables, qu'il associera notamment à différentes opérations de concertation sociale. La communication entre l'ordre professionnel et l'ordre étatique est ainsi " horizontale $\%$.

Française, l'appréciation jurisprudentielle de la représentativité à partir de critères légaux (p. 160 et suivantes) a conduit un modèle à cinq composantes confédérales, dont la suprématie se manifeste "verticalement" par voie d'une présomption de représentativité (p. 177). S'ensuit, à différents niveaux, un pouvoir normatif conféré aux partenaires sociaux, dans l'intérêt collectif de la profession, un pouvoir d'établir des conventions collectives s'imposant aux salariés indépendamment de leur volonté (p. 212).
D'une manière bien différente dans l'un et l'autre cas, la représentativité conduit à une " juridification " de l'autonomie collective. À ce titre - si une disgression d'un ouvrage à la fois si dense et déjà si ample était permise -, la comparaison engagée et son présupposé théorique feraient apparaitre la nature et l'étendue relative d'une telle juridification en contexte québécois, par exemple: pour ce qui est de la formule générale du moins, une représentativité de nature locale et effective, à laquelle les groupements peuvent généralement prétendre. Orientée vers la négociation collective d'entreprise, elle se présente comme la condition de l'effet légal erga omnes d'une telle négociation dans l'unité en cause. Une représentativité se référant à des ensemble plus vastes, à la fois plus floue et diversifiée, associe par ailleurs des groupements syndicaux à diverses fonctions étatiques, parfois dans un esprit empreint d'un certain néocorporatiste.

En second lieu, l'étude s'interroge sur la représentativité syndicale dans les rapports entre l'autonomie collective et le salarié. La convention collective envisage, rappelle-t-on, le rapport de travail comme un statut social: "loin de prévoir des dispositions orientées vers les espérances et les attentes du travailleur individuel, [elle] considère comme mesure de référence la collectivité des travailleurs, une collectivité qu'elle s'efforce à construire selon une figure neutre, le "travailleur ", dans son abstraction " (p. 228). De là, son effet impératif et automatique en droit français, les clauses du contrat individuel étant remplacées par celles de la convention collective, sauf si elles sont plus favorables (C.t., L. 135-2). L'article 2113 du Code civil italien déclare pour sa part annulable après demande du travailleur les transactions et les renonciations ayant pour objet des droits individuels découlant notamment des accords collectifs (p. 226). La représentativité du syndicat signataire de la convention collective justifie bien la portée générale de cette dernière. "Mais si l'autonomie 
individuelle se replie pour mieux résister à l'isolement du travailleur et à l'omnipotence de l'employeur, que se passe-til lorsque l'action collective cesse d'unifier et commence à diviser les travailleurs en gagnants et perdants, lorsque le travailleur ne doit plus etre protégé contre l'employeur mais contre la collectivité à laquelle il appartient " (p. 229) [ ?]

Il y va de la justification, dans l'un et l'autre droit, de la pratique de plus en plus contemporaine de la négociation dite "de gestion", des accords de solidarité, défensifs ou offensifs, favorables à l'emploi, mais emportant, par exemple des diminutions de rémunération pour des salariés en Italie, ou encore, en France, des accords dérogatoires ou, plus généralement, du développement des accords " donnant-donnant ». Certes, l'autonomie collective ne pourra disposer que des droits des travailleurs qui ne sont pas " indisponibles par nature", comme le seraient par exemple ceux leur résultant d'une loi impérative. L'analyse conduit ici à envisager la notion d'avantages individuels acquis. Mais, une seconde condition s'ajoute: la légitimité des organisations syndicales appelées à s'engager ainsi dans une telle négociation de gestion. Leur représentativité légitime-t-elle l'édiction de normes contraignantes emportant renonciation à des droits individuels des salariés? Une réponse négative parait s'imposer, puisque le « concept de représentativité se dissocie de l'idée de représentation ", pose-t-on (p. 341), après analyse (pages 300 et suivantes). Le recours au procédé référendaire apporterait un correctif approprié, de suggérer l'auteure.

Le caractère "donnant-donnant " est un caractère typique de la négociation collective nord-américaine, s'il était à nouveau permis d'extrapoler. Mais, alors représentativité et représentation légale se confondent et ont précisément pour fondement la volonté majoritaire au sein de la collectivité des salariés en cause.

L'évocation, si incomplète, du contenu de l'ouvrage, "dont la richesse ne nuit jamais à la rigueur de la construction ", comme le dit avec on ne peut plus de justesse le professeur Antoine Lyon-Caen dans sa préface, aura voulu signaler la grande portée de l'analyse des institutions essentielles des rapports collectifs du travail à laquelle il nous convie.

PIERRE VERGE

Université Laval

\section{Conflits du travail, changement social et politique en France depuis 1950}

par Monique BORREL, Paris: L'Harmattan, 1996, 266 p., ISBN 2-7384-4429-5.

À la façon de Shorter et Tilly (Strike in France: 1830-1968, Cambridge: Cambridge University Press, 1974) et plus récemment de Franzosi (The Puzzle of Strike: Class and State Strategies in Postwar Italy, Cambridge: Cambridge University Press, 1995), l'auteure veut comprendre les conflits du travail à travers une vision historique de l'évolution de la situation sociopolitique française. Cette recension porte sur son traitement de la notion de conflits du travail puisque c'est là son sujet principal. Une recension privilégiant une approche plus sociologique serait aussi profitable pour aborder les chapitres traitant de la notion de conflit, de politiques sociales ou de mobilisation syndicale. La présentation de cette recension se calque sur la démarche de l'auteure. Le livre se divise en trois parties.

La première partie "La reconstitution des séries statistiques de conflits du travail " introduit d'abord une contribution originale de l'auteure qui classifie les grèves selon leur ampleur en trois grandes catégories : (1) Les conflits localisés, c'est-à-dire les luttes de nature économique, limitées dans le temps et l'espace (l'entreprise ou l'établissement) visant à 